\section{Eat yourself for success}

Two recent papers have provided further details on the role of autophagy as an innate immune defence mechanism against bacterial pathogens.

Autophagy — literally 'self-eating' - is the process by which eukaryotic cells remove defective or excess organelles and recycle the constituent macromolecules in times of stress. A double-membrane-bound structure known as the autophagosome forms de novo and, after formation, fuses with endosomal vesicles, creating intermediate autophagosomes that go on to fuse with lysosomes, forming mature autolysosomes that can degrade their contents. Autophagy has previously been recognized as an effective method of defence against some intracellular pathogens including Listeria monocytogenes. Now, new research proves that autophagy can also help clear group A Streptococcus (GAS) and Mycobacterium tuberculosis infections.

In the first study, published in Science, Nakagawa et al., using the autophagosome-specific marker LC3 tagged with GFP, found that, in HeLa cells, intracellular GAS localized in autophagosome-like compartments. Quantitative analysis of this localization within a second cell line, embryonic stem (ES) cells — notably, like HeLa cells, these are non-phagocytic — showed that $\sim 80 \%$ of GAS were found within these compartments. The fact that the levels of the autophagosome-specific form of LC3 increased after infection suggested that the autophagy being observed was induced, and this was confirmed by analysing cells deficient in autophagosome formation. Additionally, the results obtained suggested that GAS were killed within these autophagosome-like structures.

In the second study, published online in Cell, Gutierrez et al. have shown that autophagy can inhibit the survival of $M$. tuberculosis within infected macrophages. The induction of autophagy by physiological or pharmacological signals was shown to induce increased acidification and subsequent maturation of mycobacterial-containing phagosomes, resulting in intracellular killing of the mycobacteria. Additionally, these researchers found that autophagy was also stimulated by IFN- $\gamma$, thus shedding light on the anti-mycobacterial action of this cytokine, which until now has not been fully understood. The small cytoplasmic GTPase LRG-47 was also identified as being involved in the IFN- $\gamma$-dependent induction of autophagy.

Some bacterial pathogens have evolved strategies to avoid or subvert autophagic destruction. These latest results suggest that for GAS and M. tuberculosis, in certain circumstances autophagy can be a useful addition to the host innate immune armamentarium.

Sheilagh Molloy

(2) References and links ORIGINAL RESEARCH PAPERS Nakagawa, I. et al. Autophagy defends cells against invading Group A Streptococcus. Science 306, 1037-1040 (2004) | Gutierrez, M. G. et al. Autophagy is a defense mechanism inhibiting BCG and Mycobacterium tuberculosis survival in infected macrophages. Cell 18 Nov 2004 (doi:10.1016/S0092867404011067) FURTHER READING Kirkegaard, K., Taylor, M. P. \& Jackson, W. T. Cellular autophagy: surrender, avoidance and subversion by microorganisms. Nature Rev. Microbiol. 2, 301-314 (2004)

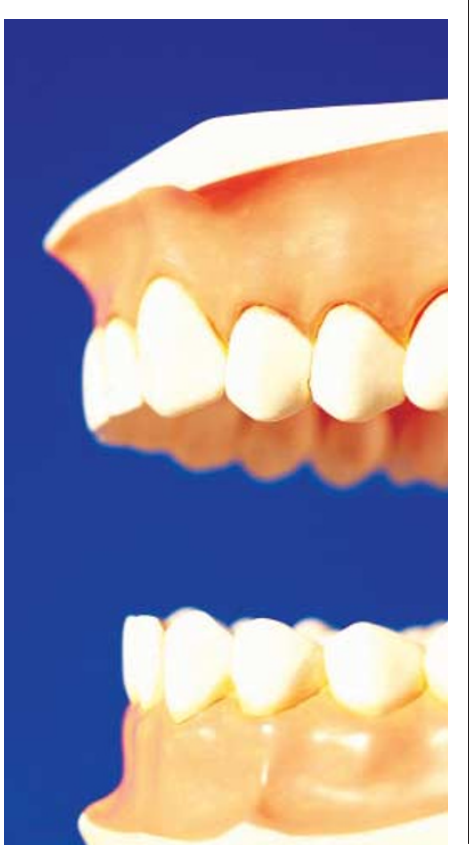

\section{IN BRIEF}

TECHNIQUES AND APPLICATIONS

\section{Real-time PCR quantitation of Clostridia in feces of} autistic children

Song, Y. et al. Appl. Environ. Microbiol. 70, 6459-6465 (2004)

In the latest issue of Applied and Environmental Microbiology, Song et al., building on previous culture-based studies, describe the development of quantitative PCR assays that can be used to quantify Clostridia clusters in stool samples. The authors designed Clostridia phylogenetic-cluster-specific oligonucleotide probes and PCR primers, and used stringent conditions to avoid amplification of unwanted sequences from closely related species. The fluorescent signal from the PCR was correlated with cell density using a platecount assay, for which careful standard curves were selected. The assay is fast, sensitive and reliable, with $>99 \%$ reproducibility.

\section{ENVIRONMENTAL MICROBIOLOGY}

\section{Tuber magnatum Pico, a species of limited} geographical distribution: its genetic diversity inside and outside a truffle ground

Mello, A. et al. Environ. Microbiol. 19 Nov 2004 (doi:10.1111/j.14622920.2004.00678.x)

The white truffle of Alba, or Tuber magnatum Pico, is the mostprized find of the trifolau scouring the hills around Alba, Italy. T. magnatum Pico is unlike other Tuber species as it has a limited geographical distribution. In this work, the genetic variability in 42 T. magnatum Pico fruiting bodies that had been collected from a natural truffle ground over a period of five years was studied. Fruiting bodies from different geographical regions were used for comparative analysis. Single-nucleotide polymorphisms were identified using the single-locus markers ITS and $\beta$-tubulin, and sequenced-characterized amplified regions (SCAR). These results, together with karyological analysis, suggest that T. magnatum can self-fertilize. Further information on the truffle research community can be found at http://www.truffle.org.

\section{ENVIRONMENTAL MICROBIOLOGY}

\section{The gut microbiota as an environmental factor that} regulates fat storage

Bäckhed, F. et al. Proc. Natl Acad. Sci. USA 101, 15718-15723 (2004)

Are bacteria making us fat? Bäckhed et al. compared the body fat content of gnotobiotic mice raised in a germ-free environment (GF); gnotobiotic mice colonized by microbiota at birth (CONV-R); and gnotobiotic mice raised in a germ-free environment until adulthood then colonized with microbiota (CONV-D). The results showed that CONV-D mice and CONV-R mice contained 57\% and $47 \%$ more body fat compared with GF mice, respectively, despite the fact that the colonized mice were fed a reduced chow diet. Increases in fasting glucose and insulin levels were also found in CONV-D mice compared with GF mice, along with increased levels of circulating leptin. Analysis showed that the presence of the microbiota increases the absorption of monosaccharides from the gut lumen and promotes storage of triglycerides in fat cells by suppressing the expression of Fiaf, an inhibitor of lipoprotein lipase. 